\title{
Automation of Datamorphic Testing
}

\author{
Hong Zhu, Ian Bayley \\ School of Engineering, Computing and Mathematics \\ Oxford Brookes University \\ Oxford OX33 1HX, UK \\ Email: (hzhu|ibayley)@ brookes.ac.uk
}

\author{
Dongmei Liu, Xiaoyu Zheng \\ School of Computer Science and Engineering \\ Nanjing University of Science and Technology \\ Nanjing 210094, China \\ Emails:dmliukz@njust.edu.cn, zxy961120@sina.com
}

\begin{abstract}
This paper presents an automated tool called Morphy for datamorphic testing. It classifies software test artefacts into test entities and test morphisms, which are mappings on testing entities. In addition to datamorphisms, metamorphisms and seed test case makers, Morphy also employs a set of other test morphisms including test case metrics and filters, test set metrics and filters, test result analysers and test executers to realise test automation. In particular, basic testing activities can be automated by invoking test morphisms. Test strategies can be realised as complex combinations of test morphisms. Test processes can be automated by recording, editing and playing test scripts that invoke test morphisms and strategies. Three types of test strategies have been implemented in Morphy: datamorphism combination strategies, cluster border exploration strategies and strategies for test set optimisation via genetic algorithms. This paper focuses on the datamorphism combination strategies by giving their definitions and implementation algorithms. The paper also illustrates their uses for testing both traditional software and AI applications with three case studies.
\end{abstract}

\section{Introduction}

With the rapid growth of artificial intelligence (AI) in computer applications, ensuring the quality of software components that employ AI techniques becomes indispensable to software engineering. However, testing AI applications is notoriously difficult and prohibitively expensive [10]. It is highly desirable to advance software test automation techniques that meet the requirements of testing AI applications.

Datamorphic testing has been proposed recently as an approach to software test automation [30]. In this method, test automation focuses on the development and application of three types of test code. Seed makers generate test cases.
Datamorphisms transform existing test cases into new ones. Metamorphisms assert the correctness of test cases. Experiments [30, 31, 19, 3] have demonstrated that it is effective at testing $\mathrm{AI}$ applications.

However, while datamorphic testing activities can be automated by writing project specific test code, it is highly desirable to develop a general testing tool to achieve the following requirements of test automation.

1. Reusibility of the test code of datamorphisms, metamorphisms, seed makers, etc, to be reused even across different projects.

2. Composability of test code in different combinations to conduct different experiments with the software under test.

3. Constructability of users' own test automation processes from existing test code so that the testing process can be repeated.

To achieve these goals, this paper extends the datamorphic testing framework by introducing the notion of test morphisms and presents an automated test tool called Mor$p h y^{1}$. It enables test automation at three levels. At the lowest level, various test activities can be performed by invoking test morphisms via a click of buttons on Morphy's GUI. At the medium level, Morphy implements various test strategies to perform complicated testing activities through combinations and compositions of test morphisms. At the highest level, test processes are automated by recording, editing and replaying test scripts that consist of a sequence of invocations of test morphisms and strategies.

The paper is organised as follows. Section 2 extends the datamorphic testing framework. Section 3 presents the Morphy test tool. Section 4 defines a set of strategies that combines datamorphisms to generate test data. Section 5 reports three case studies to demonstrate the uses of Morphy. Section 6 concludes the paper by a comparison with related work and a discussion of future work.

\footnotetext{
${ }^{1}$ Available at https://github.com/hongzhu6129/MorphyExamples.git
} 


\section{Extended Datamorphic Testing Framework}

We extend datamorphic testing method by classifying the software artefacts involved in software testing into two kinds: entities and morphisms.

Test entities are objects and data used and/or generated in testing, which include test cases, test suites, the program under test, and test reports, etc.

Test morphisms are mappings between entities. They generate and transform test entities to achieve testing objectives. They can be implemented by writing test code. They can be invoked to perform test activities and composed to form test processes. Obviously, datamorphisms, metamorphisms and seed makers in the existing model of datamorphic testing are all test morphisms. However, there are other types of test morphisms that play crucial roles in test automation.

A software test specification in this extended framework specifies both of these artefacts and enables them to be invoked, composed as well as reused as a test library. In Morphy, a test specification is a Java class that declares a set of attributes for test entities and a set of methods for test morphisms.

In this section, we discuss how they are defined in order to meet the requirements of test automation.

\subsection{Test Entities}

Test cases and test suites are the most important kinds of entities on which test morphisms are defined. To enable the definition of various test morphisms, a test case must contain not only information about the input and output of the software, but also information about the following:

- How the test case is generated. Two particular pieces of information about the test case are recorded: whether it is a seed or a mutant, and which test morphism generates the test case. In the sequel, the former is called the feature of the test case, the latter is called the type of the test case.

- How a test case is related to other test cases. If a test case is generated by using a datamorphism, the identities of test cases on which the datamorphism applied are recorded, and they are called the origins of the test case.

- The correctness of the test case. In datamorphic testing, the correctness of a test case is checked against metamorphisms. Each metamorphism can be a partial correctness condition. Therefore, test case may pass some of the metamorphisms but fail on the others. Therefore, the correctness of a test case is a set of records of checking the test case against metamorphisms. We will use the following format to record the correctness:

$$
\{\text { metamorphismName: (pass } \mid \text { fail })\} *
$$

A test suite consists of a list of test cases. Each test case is also assigned with a universally unique identifier (UUID). Therefore, the relationships between test cases can be defined by references to their UUIDs.

The Morphy testing tool defines two generic classes TestCase and TestPool for representing test cases and test suites, respectively. They have two type parameters for the input and output datatypes.

The generic class TestCase consists of attributes for (a) the UUID of the test case, (b) the input data, (c) the output data, (d) the feature, (e) the type of the test case, (f) the list of origins, and $(\mathrm{g})$ the correctness of the test case.

The generic class TestPool consists of a list of TestCases and a number of methods for the operations of the test suite, such as adding and removing test cases to/from the test suite. The test suite used in the testing of the software is declared as an attribute of TestPool type and annotated with metadata @TestSetContainer. A test specification class can also have attributes and methods without annotations. For examples, an attribute of TestPool type without annotation @ TestSetContainer can be used as an auxiliary test set.

The source code of the TestCase and TestPool can be found in [29].

\subsection{Test Morphisms}

In addition to the three components of the original datamorphic testing model, we identify the following types of test morphisms that are useful to automate software testing.

- Test case metrics are mappings from test cases to real numbers. They measure test cases, for example, on the similarity of a test case to the others in the test set.

- Test case filters are mappings from test cases to truth values. They can be used, for example, to decide whether a test case should be included in the test set.

- Test set metrics are mappings from test sets to real numbers. They measure the test set, for example, on its quality, such as code coverage.

- Test set filters are mappings from test sets to test sets. A typical example is to remove some test cases from a test set for regression testing.

- Test executers execute the program under test on test cases and receive the outputs from the program. They are mappings from a piece of program to a mapping from input data to output. That is, they are functors in category theory.

- Test result analysers analyse test results and generate test reports. Thus, they are mappings from test set to test reports. 


\subsection{Test Specifications}

A Morphy test specification is a Java class, which declares a set of attributes as test entities and a set of methods as test morphisms; see [29] for an example. Each test morphism is annotated with a metadata to declare the type of test morphism that the method belongs to. Table 1 lists the annotations and datatypes of various types of test morphisms as implemented in Morphy.

Table 1. Annotations of Test Morphisms

\begin{tabular}{||l|l|l|l||}
\hline \hline Morphism & Annotation & Parameter & Return \\
\hline \hline Seed Maker & @ SeedMaker & Nil & Void \\
\hline Datamorphism & @ Datamorphism & TestCase & TestCase \\
\hline Metamorphism & @ Metamorphism & TestCase & Boolean \\
\hline Test Case Metrics & @ TestCaseMetrics & TestCase & Real \\
\hline Test Case Filter & @ TestCaseFilter & TestCase & Boolean \\
\hline Test Set Metrics & @ TestSetMetrics & Nil & Real \\
\hline Test Set Filter & @ TestSetFilter & Nil & Nil \\
\hline Test Executer & @ TestExecuter & Input & Output \\
\hline Analyser & @ Analyser & Nil & Void \\
\hline \hline
\end{tabular}

\section{Test Tool Morphy}

As shown in Figure 1, Morphy consists of three main facilities: test set management, test runner and test scripting.

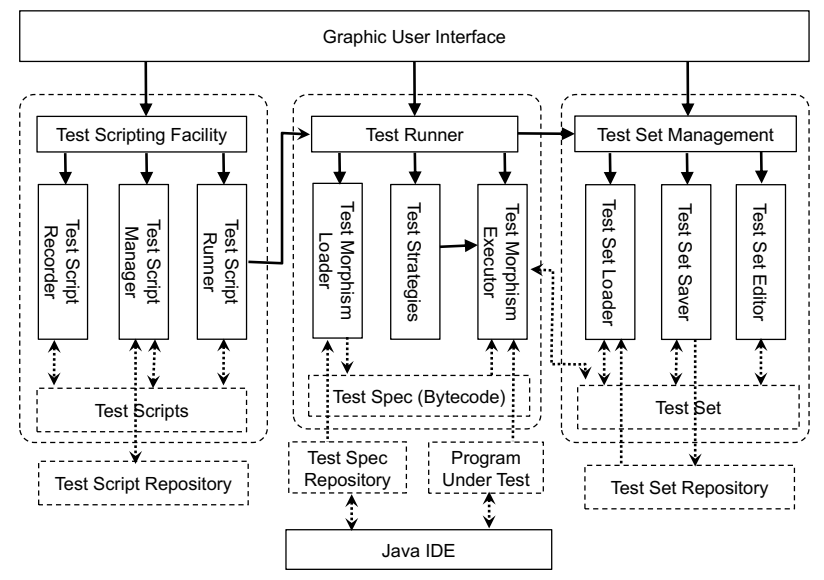

Figure 1. The Architecture of Morphy

The test set management facility enables test sets to be saved into files, loaded from files and edited in a graphic user interface. The test runner enables test specifications to be loaded into the system and various test morphisms of the test specification to be invoked. It also implements various test strategies. The test scripting facility enables interactive testing activities to be recorded as test scripts, saved into files, reloaded from files and replayed.

Test specifications can be developed with any Java IDE, but a wizard has been developed as an Eclipse plugin to generate new skeleton Java class of test specification.

Morphy's main graphic user interface shown in Figure 2 provides a user friendly environment in which testing artefacts can be managed, basic testing activities can be performed and automated testing facilities can be invoked.

At the very the top of Morphy's main window are four panels of buttons for the management of test entities, performing test activities by invoking various types of test morphisms, applying test strategies, and recording-replaying test scripts, respectively.

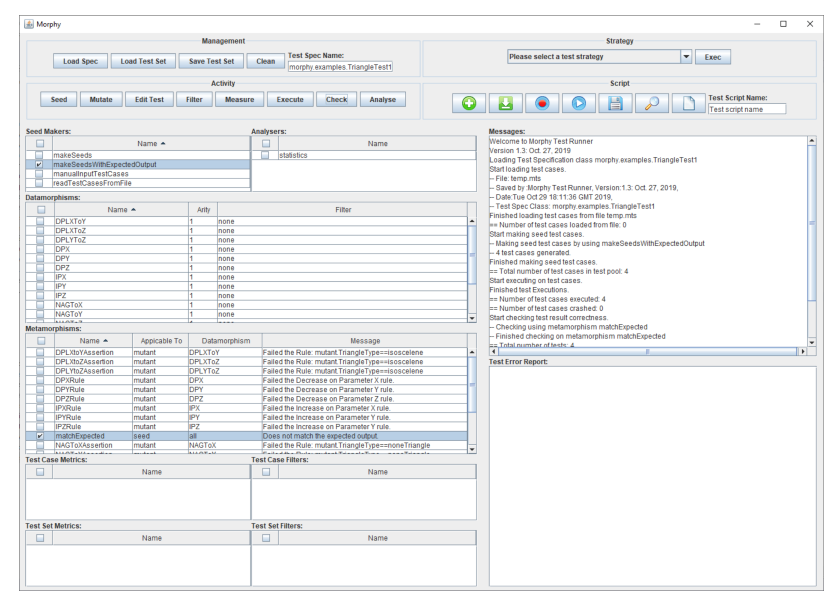

Figure 2. Morphy's Main GUI

Three sets of test strategies have been implemented in Morphy:

- Mutant combination: combining datamorphisms to generate mutant test cases;

- Domain exploration: searching for the borders between clusters/subdomains of the input space;

- Test set optimisation: optimising test sets by employing genetic algorithms.

Due to the limitation of space, this paper focuses on the mutant combination strategies, which is given in Section 4. The other two types of test strategies will be reported separately.

The test script facility allows the user to automate the testing process. It is particularly useful for repeated testing processes, such as in regression testing and repeated experiments with the software under test to obtain data for statistical analysis.

The left-hand side of the main window is a list of tables that shows various types of test morphisms. The elements in these tables can be selected by clicking on the check boxes on the first column as input to perform the interactive and automatic testing functions.

The right-hand side contains two message panels. The upper one reports the testing activities performed and their outcomes. The lower report errors detected by checking the test results against metamorphisms. 


\section{Mutant Combination Strategies}

Let $T$ be the set of all possible test cases for the software under test. $S \subset T$ be a set of test cases. $D$ be a set of datamorphisms and $d \in D$ is a datamorphism in $D$. We say that $d$ is $k$-ary $(k>0)$, if $d: T^{k} \rightarrow T$.

\section{Definition 1 (First Order Mutants)}

A test case $y \in T$ is called a first order mutant test case, or simply a first order mutant, of $S$ generated by $D$, if there is a $k$-ary datamorphism $d \in D$ and test cases $x_{1}, \cdots, x_{k} \in$ $S$ such that $y=d\left(x_{1}, \cdots, x_{k}\right)$.

A set $C$ of test cases is first order mutant complete with respect to $S$ and $D$, if $S \subseteq C$, and for each $d: T^{k} \rightarrow T \in$ $D$, and each $x_{i} \in S, i=1, \cdots, k$, there is a test case $y \in C$ such that $y=d\left(x_{1}, x_{2}, \cdots, x_{k}\right)$, where $d$ is $k$-ary.

In other words, a test set is first order mutant complete if it contains every seed and every first order mutant. A test strategy is to test the software with all the seeds and all the first order mutant test cases generated from the seeds using selected datamorphisms.

The following algorithm generates the minimal test set that is first order mutant complete with respect to a give set of seed test cases and a set of datamorphisms.

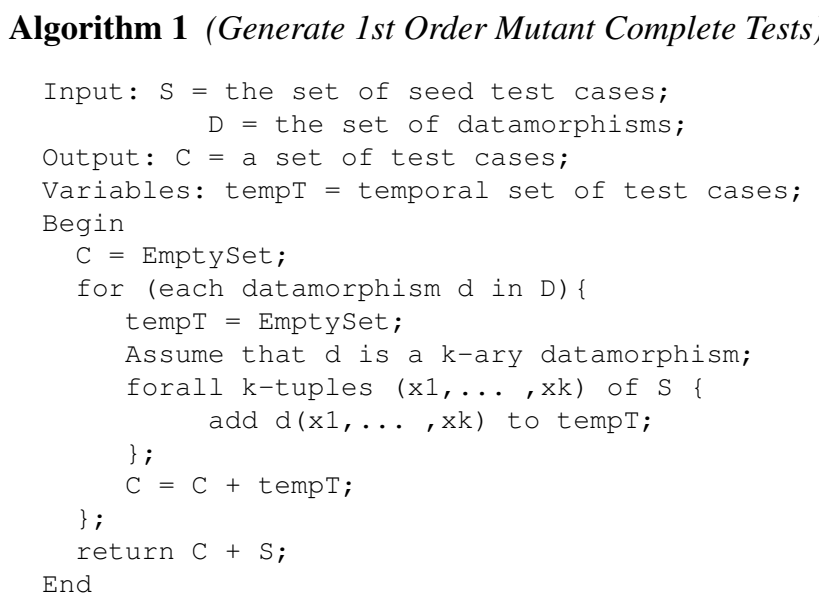

The following theorem asserts the correctness of the algorithm. The proof can be found in [29].

Theorem 1 The test set generated from $S$ using $D$ by Algorithm 1 is the minimal set of test cases that is first order mutant complete with respect to $S$ and $D$.

For example, consider a software system that takes a point in the two-dimensional space of real numbers and classifies the points into three subdomains: the red, the blue and the black areas. The test set initially contains 100 random points. The datamorphism is to add the middle point of two test cases. Applying Algorithm 1 produces a first order mutant complete test set, which contains 10000 test cases. Figure 3 (a) and (b) below shows the results of testing on the

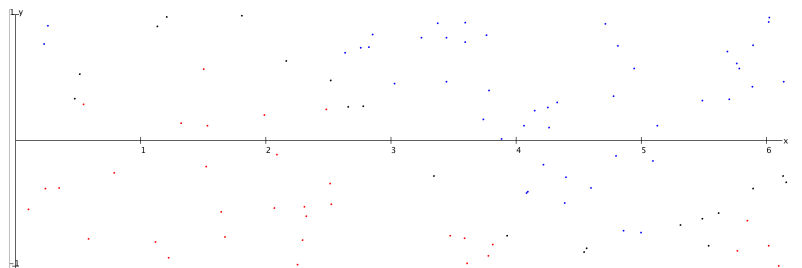

(a) Original Test Set

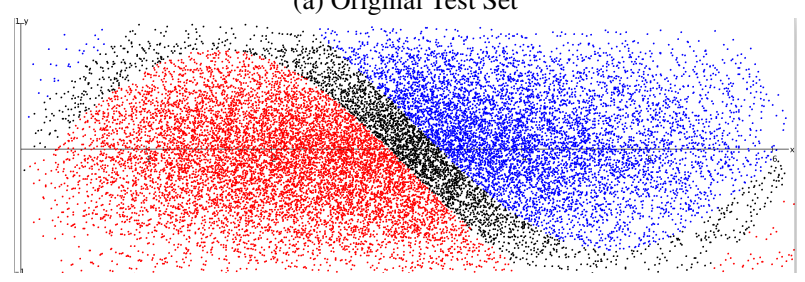

(b) 1 st Order Mutant Complete Test Set

Figure 3. Test Results

original 100 random test cases and on the 1st order mutant complete test set, respectively.

Datamorphisms can also be applied to test cases multiple times to generate mutants of mutants, which are called high order mutants. For the sake of convenience, a test case $x \in$ $S$ is called a 0 'th order mutant of $S$.

Definition 2 (Higher order mutants)

A test case $y$ is a second order mutant of $S$ by $D$, if there is a $k$-ary datamorphism $d \in D$ and $k$ test cases $x_{1}, \cdots, x_{k}$ such that $y=d\left(x_{1}, \cdots, x_{k}\right)$ and for all $x_{i}, x_{i}$ is either in $S$ or a first order mutant of $S$ by $D$, and at least one of $x_{1}, \cdots, x_{k}$ is a first order mutant of $S$ by $D$.

A test case $y$ is an $n$ 'th order mutant of $S$ by $D(n>1)$, if there is a $k$-ary datamorphism $d \in D$ and $k$ test cases $x_{1}, \cdots, x_{k}$ such that $y=d\left(x_{1}, \cdots, x_{k}\right)$ and $x_{i}$ are $m$ 'th order mutants of $S$ by $D$, where $m<n$, and at least one of $x_{1}, \cdots, x_{k}$ is a $(n-1)$ 'th order mutant of $S$ by $D$.

Similar to first order mutant completeness, a test set is 2nd order mutant complete if it contains all seed test cases, all 1 st order mutants and all 2 nd order mutants. In general, we have the following definition.

Definition 3 ( $K$ 'th order mutant completeness) A set $C$ of test cases is k'th order mutant complete with respect to $S$ and $D$, if it contains all $i$ 'th order mutant test cases of $S$ by $D$ for all $i=0, \cdots, k$.

The following can be proved based on Theorem 1 by induction on the order $K$.

Corollary 1 of Theorem 1. By repeating Algorithm 1 for $K$ times that each time uses the output test set as the input to the next invocation of the algorithm, the result test set is the minimal K'th order mutant complete.

Assume that the set $D$ of datamorphisms contains $N$ methods. If a test set is $N$ 'th order mutant complete with 
respect to $S$ and $D$, it contains all permutations of the datamorphisms applied to all test cases. We say that the test set is permutation complete. If the datamorphisms are associative, commutative, distributive and idempotent, a permutation complete test set contains all possible test cases that can be derived from a give set of test cases using the set of datamorphisms. The test set is therefore exhaustive with regard to the set of seeds and the datamorphisms. It usually contains a huge number of test cases, so the cost of testing can be very high. A compromise is to cover the combinations of datamorphisms.

A mutant of $S$ by $D$ can be represented as a tree on which the leaf nodes are test cases in $S$, and the non-leaf nodes are datamorphisms in $D$. The order of a mutant is the height of the tree. Figure 4 below shows some examples of mutants, in which (a) and (b) are first order mutants, and (c) to (f) are second order mutants.

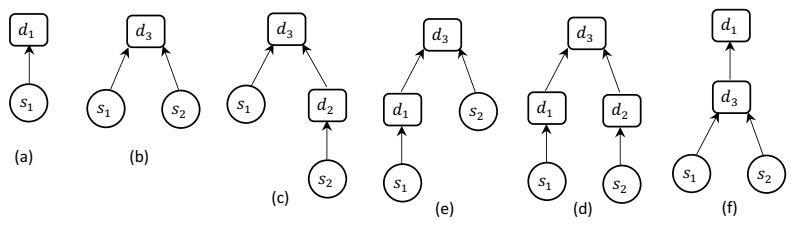

Figure 4. Examples of Mutant Trees

Given a mutant's tree representation, by replacing the test cases associated to the leaf nodes with variables in such a way that each different leaf node is associated with a different variable that ranges over the test cases, we can then obtain a function that generates a high order mutant when substitutes the variables with seed test cases. Each tree of this kind is therefore a way to combine datamorphisms to make higher order mutant test cases from seed test cases. We say that a combination $c$ is $k$-ary, if it contains $k$ variables, which is equivalent to the number of leaf nodes. We write $c\left(x_{1}, \cdots, x_{k}\right)$ to represent such a combination of datamorphism. When applying $c$ to seed test cases $a_{1}, \cdots, a_{k}$, we write $y=c\left(a_{1}, \cdots, a_{k}\right)$ to denote the result mutant test case. Let $\left\{d_{1}, \cdots, d_{v}\right\}$ be the set of datamorphisms in the tree, we also say that $c$ is a combination of $\left\{d_{1}, \cdots, d_{v}\right\}$. Given a set $D$ of datamorphisms, there may be many different combinations of $D$.

Definition 4 (Complete set of datamorphic combinations)

A set $C$ of datamorphism combinations is combinatorial complete for $D$, if for all subsets $D^{\prime} \subseteq D$, there is a combination $c \in C$ that contains exactly the datamorphisms in $D^{\prime}$.

A set $V$ of test cases is combinatorial complete with respect to $S$ and $D$, if

- there is a set $C$ of datamorphism combinations that is combinatorial complete with respect to $D$; and
- for every combination $c \in C$, if $c$ is $k$-ary, then for all $k$-tuple of test cases $\left(x_{1}, \cdots, x_{k}\right) \in S^{k}$, there is a test case $y$ in $V$ such that $y=c\left(x_{1}, \cdots, x_{k}\right)$.

The following is an algorithm that generates a combinatorial complete test set.

Algorithm 2 (Generate Combinatorial Complete Test Set)

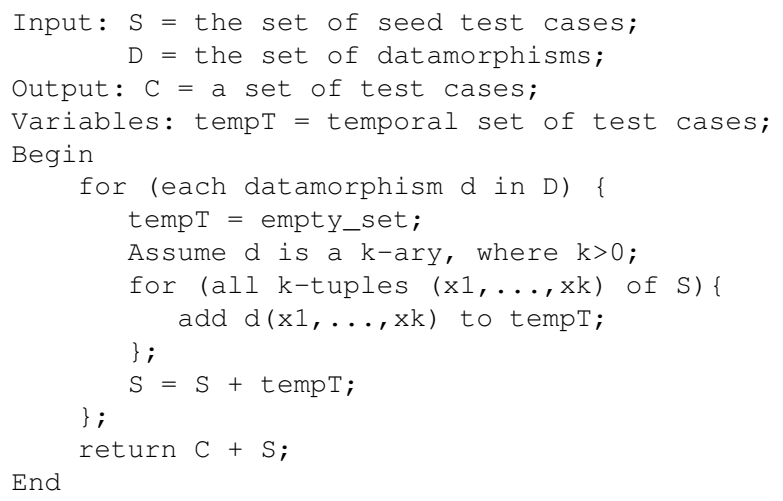

Theorem 2 The test set generated by Algorithm 2 is combinatorial complete with respect to $S$ and $D$.

Note that, the test set generated by Algorithm 2 may be not minimal in size if there is a datamorphism that is nonunary.

\section{Case Studies}

We have conducted three case studies on the development of Morphy test specifications and the uses of Morphy in automated software testing. ${ }^{2}$ These case studies are:

\section{- Triangle Classification.}

Triangle classification is a classic software testing problem that Myer used to illustrate the importance of combination of various types of test cases [20]. The program under test "reads three integer values from an input dialog. The three values represent the lengths of the sides of a triangle. The program displays a message that states whether the triangle is scalene, isosceles, or equilateral." [20] Myer listed 14 questions for testers to assess the adequacy of a test and reported that, for such a seemly simple program, "highly qualified professional programmers score, on the average, only 7.8 out of a possible 14 ".

The case study demonstrated that datamorphisms can be easily developed to achieve test adequacy and the testing process can be automated. A set of 20 datamorphisms were developed inspired by Myer's test criteria. When the first

\footnotetext{
${ }^{2}$ The source code of the case studies can be found on GitHub at the URL: https://github.com/hongzhu6129/MorphyExamples.git
} 
order mutant complete strategy is applied to these datamorphisms on 4 seed test cases, 80 mutant test cases are generated automatically, which fully meet Myer's test criteria. Moreover, for each datamorphism, we also developed a corresponding metamorphism to check the correctness of the program under test. Four different programs were tested: two are incorrect and two are correct but using different algorithms. The testing successfully detected the bugs in the faulty programs, while the correct ones passed the test. The test specifications were split into two classes, thus test morphisms were reused; see Figure 5.

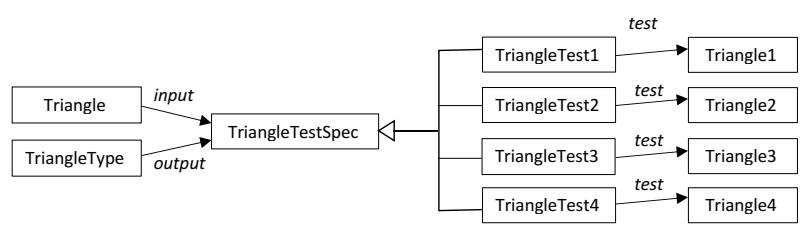

Figure 5. Structure of Test Specification

\section{- Trigonometric Functions.}

Three trigonometric functions $\sin (x), \cos (x)$ and $\tan (x)$ provided by Java Math library are tested. The correctness of the library's implementation of these functions are checked against a set of 27 trigonometric identities implemented as metamorphisms; see Table 2.

Two seed makers were written: one generates a set of 17 special values between 0 and $2 \pi$; the other generates 100 random test cases in the range between 0 and $\frac{\pi}{2}$.

Unary datamorphisms were written for the mappings from $x$ to $2 \pi \pm x, \pi \pm x, \frac{\pi}{2} \pm x,-x$, and binary datamorphisms from $x$ and $y$ to $x \pm y$, and $\frac{x+y}{2}$.

The first order mutant complete and the combination complete test sets were generated using Morphy testing tool. The testing revealed an error rate of $0.957 \%$, which are on test cases that the inputs to $\tan (x)$ are not defined, or very close to undefined.

\section{- Face Recognition.}

The experiments with face recognition reported in [30, 31] are repeated, but the test code is re-written in the form of Morphy test specifications. The case study clearly demonstrated the benefit of test automation and the reusability of test code achieved by Morphy. The test data for testing a face recognition application are images of sizes more than $100 \mathrm{~KB}$. In [30, 31, 19], 200 images of different persons were used and each generated 13 mutants using AttGAN [13] to alter the facial features. In the case study, each mutant image was only generated once and stored in the file system, then it was reused to test different face recognition applications rather than generated many times.
Table 2. List of Metamorphisms

\begin{tabular}{ll}
$\sin (\pi-x)=\sin (x)$ & $\sin (\pi+x)=-\sin (x)$ \\
$\cos (\pi-x)=-\cos (x)$ & $\cos (\pi+x)=-\cos (x)$ \\
$\tan (\pi-x)=-\tan (x)$ & $\tan (\pi+x)=\tan (x)$ \\
$\sin (\pi / 2+x)=\cos (x)$ & $\sin (\pi / 2-x)=\cos (x)$ \\
$\cos (\pi / 2+x)=-\sin (x)$ & $\cos (\pi / 2-x)=\sin (x)$ \\
$\tan (\pi / 2+x)=-1 / \tan (x)$ & $\tan (\pi / 2-x)=1 / \tan (x)$ \\
$\sin (2 \pi-x)=-\sin (x)$ & $\sin (2 \pi+x)=\sin (x)$ \\
$\cos (2 \pi-x)=\cos (x)$ & $\cos (2 \pi+x)=\cos (x)$ \\
$\tan (2 \pi-x)=-\tan (x)$ & $\tan (2 \pi+x)=\tan (x)$ \\
$\sin (-x)=-\sin (x)$ & $\cos (-x)=\cos (x)$ \\
$\tan (-x)=-\tan (x)$ & \\
$\sin (x+y)=\sin (x) \cos (y)+\cos (x) \sin (y)$ \\
$\cos (x+y)=\cos (x) \cos (y)-\sin (x) \sin (y)$ \\
$\sin (x-y)=\sin (x) \cos (y)-\cos (x) \sin (y)$ \\
$\cos (x-y)=\cos (x) \cos (y)+\sin (x) \sin (y)$ \\
$\tan (x+y)=(\tan (x)+\tan (y)) /(1-\tan (x) \tan (y))$ \\
$\tan (x-y)=(\tan (x)-\tan (y)) /(1+\tan (x) \tan (y))$ \\
\hline
\end{tabular}

In addition to repeating the previous experiments, which examines whether a face recognition application recognises a person from a mutant image, a new experiment was designed to examine whether a face recognition application rejects a mutant images of a different person. The new experiment is implemented by writing just one new seed maker. All other test morphisms are reuses of the existing ones. The test specifications are split into three classes: one for datamorphisms and analysers, one for seed makers and one for test executer. This is in the similar structure to the test specifications for Triangle Classification.

Test scripts were recorded and slightly edited to add code for repeating tests for a number of times in order to obtain statistically significant data. A test analyser method was also written to do statistical analysis of the experiment data. The test process was highly automated and repeatable.

Table 3. Summary of Case Studies

\begin{tabular}{|l|c|c|c|}
\hline \hline & TC & Trg & FR \\
\hline \hline Num of Classes & 11 & 4 & 8 \\
\hline Total LOC & 899 & 830 & 450 \\
\hline Num of Seed Makers & 4 & 3 & 3 \\
\hline Average LOC of Seed Makers & 26.25 & 61.67 & 21.33 \\
\hline Num of Datamorphisms & 20 & 10 & 13 \\
\hline Average LOC of Datamorphisms & 9 & 6 & 8 \\
\hline Num of Metamorphisms & 25 & 30 & - \\
\hline Average LOC of Metamorphisms & 8.72 & 7.00 & - \\
\hline Num of Analysers & 2 & 2 & 2 \\
\hline Average LOC of Analysers & 62 & 33 & 41 \\
\hline \hline
\end{tabular}

The following observations were made on the case studies. First, test morphisms in the case studies are simple and easy to write; see Table 3, where TC stands for Triangle Classification, Trg for Trigonometric Function, and FR for Face Recognition. LOC is the lines of code. 
Second, test specifications are reusable especially when they are properly structured. In the case study, test specifications are decomposed into a number of classes where common test morphisms are placed together. They are inherited by classes that contain test specific morphisms.

Third, achieving test automation using facilities at three different levels of activity, strategy and process is flexible and practical. Different testing techniques can be easily integrated into Morphy and used together.

\section{Conclusion}

\subsection{Main Contributions}

The main contributions of the paper are three folds. First, this paper redefines datamorphic testing method by classifying test artefacts into test entities and morphisms. Datamorphisms, metamorphisms and seed makers are examples of test morphisms. We have also identified a set of other test morphisms, which include test case metrics and filters, test set metrics and filters, test executers and analysers. The case studies reported in this paper have clearly demonstrated the importance of the test morphisms of test executers and analysers. The other types of test morphisms also play crucial roles in the implementation of test strategies, which will be reported in separate papers.

Second, the paper proposes a novel framework of test automation and demonstrates its feasibility by a test automation tool called Morphy. In this framework, testing activities can be automated by writing test codes for various test morphisms and invoking them through a test tool like Morphy. Advanced combinations of test morphisms can be realised by test strategies to achieve a higher level of test automation. Three types of test strategies have been implemented in Morphy: (a) Datamorphism combination strategies generate test sets of various coverage of datamorphism combinations; (b) Exploration strategies explore the test space in order to find the borders between subdomains for testing classification and clustering type of AI applications; (c) Test set optimisation strategies employ genetic algorithms to optimise test sets. This paper focuses on datamorphism combination strategies. They are formally defined and their implementation algorithms are presented. Their uses are demonstrated by case studies. The other types of test strategies will be reported separately. Morphy also provides a test scripting facility to further improve test automation especially for regression testing. Test scripts can be recorded from the interactive invocations of test morphisms for basic test activities and invocations of test strategies as well as test management activities such as loading test specifications, loading and saving test sets, etc. The case studies reported in this paper used test scripts to improve test automation. A more detailed study of the test script facility will be reported in a separate paper.

Third, the paper reports three case studies with datamorphic testing method and the automated testing tool Morphy. The case studies demonstrated the practical usability of the method and the tool. In particular, Morphy is applicable to all kinds of software systems including AI applications.

\subsection{Related Work}

There are two kinds of test automation frameworks: XUnit [11, 18] like JUnit and GUI based test automation tools like Selenium [23] and WebDriver [26]. In compariosn with them, Morphy provides more advanced test automation facilities such as test strategies.

In XUnit framework test is defined by a set of methods in a class or a set of test scripts for executing the program under test together with methods for setting up the environment before test executions and tearing down the environment after test. Such a test specification is imperative. Our test specifications are declaratively imperative in the sense that each test class declares various testing morphisms while each test morphism is coded in an imperative programming language. Our case studies show that such test specifications are highly reusable and composible even for testing different applications. This is what existing test automation frameworks have not achieved.

GUI based test automation tools employs test scripts or test code to interact with GUI elements. The most representative and most well-known example of such testing tools is Selenium [23]. It has two test environments: (a) the Selenium IDE in which manual testing can be recorded into test scripts and replayed; (b) the Web Drivers, which provided an API for writing test code in programming languages. Morphy also employs test scripts, but it is equipped with more advanced test automation facilities such as test strategies, thus it achieves a higher level of test automation.

An advantage of Morphy is that the architecture enables various testing techniques and tools to be integrated by wrapping exiting testing tools as methods in a test specification to invoke the tools. For example, test case generation techniques and tools [1] like fuzz testing [25], data mutation testing [22], random testing [2], adaptive random testing $[8,17]$, combinatorial testing [21] and model based test case generators are all test morphisms, which can be wrapped as seed makers or datamorphisms. Metamorphic relations in metamorphic testing [7] and formal specification-based test oracles $[4,5,6,28,15,16]$ are metamorphisms. Test coverage measurement tools like [24] are test set metrics. Regression testing techniques and methods [27] that select or prioritise test cases in an existing test set can be implemented as test set filters. Search-based testing $[12,9]$ can be regarded as test strategies. Therefore, they can all be easily integrated into Morphy. 


\subsection{Future Work}

It is worth noting that datamorphic testing focuses on test morphisms related to test data and test sets, as its name implies. There are other types of test morphisms. For example, mutation operators in mutation testing [14] and fault injection tools for fault-based testing methods are test morphisms that are mappings from programs to programs or to sets of programs. Specification mutation operators are test morphisms that mapping from formal specifications to specifications, or to sets of specifications. It is an interesting further research question how to integrate such test morphisms into the datamorphic testing tools like Morphy, although, theoretically speaking, there should be no significant difficulty to do so.

It is also possible to integrate XUnit like JUnit and GUI based test automation tools like WebDriver with Morphy. This is also an interesting topic for future work.

We have already conducted some experiments with the exploration strategies and test set optimisation strategies. The results will be reported in separate papers.

\section{References}

[1] S. Anand, et al. An orchestrated survey of methodologies for automated software test case generation. JSS, 86(8):1978 2001, 2013.

[2] A. Arcuri, M. Z. Iqbal, and L. Briand. Random testing: Theoretical results and practical implications. IEEE TSE, 38(2):258-277, March 2012.

[3] Y. Bagge. Experiments with testing a bounded model checker for C. MSc Dissertation, School of Eng., Comp. and Math., Oxford Brookes Univ., Oxford, UK, Sept. 2019.

[4] G. Bernot, M. C. Gaudel, and B. Marre. Software testing based on formal specifications: a theory and a tool. SEJ, pp387-405, Nov. 1991.

[5] H. Chen, T. Tse, and T. Chen. In black and white: an integrated approach to class-level testing of object-oriented programs. ACM TOSEM, 7(3):250-295, 1998.

[6] H. Chen, T. Tse, and T. Chen. Taccle: a methodology for object-oriented software testing at the class and cluster levels. ACM TOSEM, 10(1):56-109, 2001.

[7] T. Y. Chen, et al. Metamorphic testing: A review of challenges and opportunities. ACM Comput. Surv., 51(1):4:14:27, Jan. 2018.

[8] T. Y. Chen, H. Leung, and I. K. Mak. Adaptive random testing. In Proc. of ASCC 2004., LNCS 3321, pp320-329. Springer, 2004.

[9] M. Dave and R. Agrawal. Search based techniques and mutation analysis in automatic test case generation: A survey. In Proc. of ACC 2015, pp795-799, June 2015.

[10] A. Gotlieb, M. Roper, and P. Zhang, eds. Proc. of The 1st IEEE Int'l Conf. on Artificial Intelligence Testing (AITest 2019). IEEE Computer Society, Apr 2019.

[11] P. Hamill. Unit Test Frameworks. O’Reilly, 2005.
[12] M. Harman, A. Mansouri, and Y. Zhang. Search based software engineering: Trends, techniques and applications. ACM Comput. Surv., 45(1):Article 11, 61 pages, Nov. 2012.

[13] Z. He, W. Zuo, M. Kan, S. Shan, and X. Chen. AttGAN: Facial attribute editing by only changing what you want. IEEE Trans. on Image Processing, 28(11):5464-5478, Nov 2019.

[14] Y. Jia and M. Harman. An analysis and survey of the development of mutation testing. IEEE TSE, 37(5):649-678, Sep. 2011.

[15] L. Kong, H. Zhu, and B. Zhou. Automated testing EJB components based on algebraic specifications. In Proc. of COMPSAC 2007(2), pp717-722, 2007.

[16] D. Liu, X. Wu, X. Zhang, H. Zhu, and I. Bayley. Monic testing of web services based on algebraic specifications. In Proc. of SOSE 2016, Oxford, UK, March 2016.

[17] Y. Liu and H. Zhu. An experimental evaluation of the reliability of adaptive random testing methods. In Proc. of SSIRI 2008, pp24-31, Yokohama, Japan, July 2008.

[18] G. Meszarros. xUnit Test Patterns: Refactoring Test Code. Addison Wesley, 2007.

[19] S. Mugutdinov. Applying datamorphic technique to test face recognition applications. BSc dissertation, School of Eng., Comp. and Math., Oxford Brookes Univ., Oxford, UK, Mar. 2019.

[20] G. J. Myers. The Art of Software Testing. John Wiley and Sons, Inc., 2nd ed., 2004.

[21] C. Nie and H. Leung. A survey of combinatorial testing. ACM Comput. Surv., 43(2):11:1-11:29, Feb. 2011.

[22] L. Shan and H. Zhu. Generating structurally complex test cases by data mutation: A case study of testing an automated modelling tool. Computer Journal, 52(5):571-588, Aug 2009.

[23] Software Freedom Conservancy. Selenium website. Online at URL: https://selenium.dev, Nov. 2019.

[24] Stackify. The ultimate list of code coverage tools. Online at URL: https://stackify.com/code-coverage-tools/, May 2017.

[25] M. Sutton, A. Greene, and P. Amini. Fuzzing: Brute Force Vulnerability Discovery. Addison-Wesley, 2007.

[26] WWW Consortium. WebDriver: W3C Recommendation. At URL: https://www.w3.org/TR/webdriver1/, June 2018.

[27] S. Yoo and M. Harman. Regression testing minimization, selection and prioritization: A survey. JSTVR, 22(2):67120, Mar. 2012.

[28] H. Zhu. A note on test oracles and semantics of algebraic specifications. In Proc. of QSIC'03, pp91-99, Dallas, USA, Nov. 2003.

[29] H. Zhu, I. Bayley, D. Liu and X. Zheng. Morphy: A datamorphic software test automation tool. Technical Report OBU-ECM-AFM-2019-01, School of Eng., Comp. and Math., Oxford Brookes Univ., Oxford, UK, Dec. 2019. URL: http://cms.brookes.ac.uk/staff/ HongZhu/Publications/MorphyTechnicalReport2019.pdf

[30] H. Zhu, D. Liu, I. Bayley, R. Harrison, and F. Cuzzolin. Datamorphic testing: A method for testing intelligent applications. In Proc. of AITest 2019, pp149-156, Apr 2019.

[31] H. Zhu, D. Liu, I. Ian Bayley, R. Harrison, and F. Cuzzolin. Datamorphic testing: A methodology for testing AI applications. Technical Report OBU-ECM-AFM-2018-02, School of Eng., Comp. and Math., Oxford Brookes Univ., Oxford, UK, Dec 2018. 